\title{
Morfología polínica de especies del género Senna (Fabaceae) del Sureste del Iberá, Corrientes, Argentina
}

\section{Lionel Fernandez-Pacella}

Universidad Nacional del Nordeste, Centro de Ecología Aplicada del Litoral, CONICET, CP 3400- Corrientes, Argentina; lionelpacella@yahoo.com.ar

\author{
Recibido 06-V-2013. Corregido 10-XI-2013. Aceptado 09-XII-2013.
}

\begin{abstract}
Pollen morphology of species of genus Senna (Fabales: Fabaceae) in Southeast Ibera, Corrientes, Argentina. The system of Ibera lake and marshes in the Province of Corrientes, covers an area of approximately $12000 \mathrm{~km}^{2}$, and is considered one of the most important wetlands in Latin America. Given the wide diversity of tropical communities in this area, it is important to generate new information about the different species of this ecosystem, through pollen morphology, as lake sediments favor the pollen grains conservation. With the aim to promote future research on systematics, vegetation history and melissopalynology of this region, this study attempted to describe the pollen morphology of the well represented genus Senna. For this, we conducted 5-8 days collection trips in Southeast Ibera, on a monthly basis between 2009 and 2011. Based on the plants collected, the literature review and databases from the herbarium of the Botanical Institute of Northeast Argentina, we developed a list of species for the Province of Corrientes. For the pollen morphology, we manually took at least three flower buds per flower in the sampled sites, and for those species not sampled in the field, samples were obtained from the IBONE herbarium. Palynological material preparation was carried out by a modified technique of Erdtman acetolysis. The palynological descriptions considered the polar and equatorial diameter, exine thickness, openings and sculptural elements. Our results showed that Senna genus is euripalynic, composed of 3-colporate pollen grains, small to medium size, radiosymmetric, isopolar, oblates-spheroidals to prolates, subcircular field, subtriangular to triangular and long colpis. We described for the first time, the pollen morphology of 17 species of the genus Senna for this area, and recognized two groups considering endoapertures: lalongates and circular. The list of species belonging to the genus Senna presented here could be considered in restoration and recovery plans. A major constraint for the correct interpretation of the environmental changes impacts in vegetation, whether of climatic and/or anthropogenic origin, is the knowledge of pollen morphology of tropical species. A palynological database is of high value for the interpretation and determination of plant species. Rev. Biol. Trop. 62 (2): 769-782. Epub 2014 June 01.
\end{abstract}

Key words: Senna pollen morphology, Southeast Ibera, diversity, tropical communities, paleoecological, Caesalpinioideae.

Dada la amplia diversidad de las comunidades tropicales en la Provincia de Corrientes, es importante documentar a través de floras polínicas, la morfología de las distintas especies que las conforman y que sirvan de base para futuras investigaciones sobre sistemática, historia de la vegetación y melisopalinología de esta región.

En la Provincia de Corrientes, el sistema de esteros y lagunas del Iberá, abarca aproximadamente $12000 \mathrm{~km}^{2}$ de superficie y es considerado uno de los humedales más importantes de Latinoamérica. Por su extensión se halla en el decimoquinto lugar entre las planicies anegables e inundables de Sudamérica (Neiff, 1997). En general, puede expresarse que corresponde a una dilatada planicie muy escasa que se orienta hacia el S, y cuya somera cubeta es alimentada fundamentalmente por las lluvias (1200 a $1500 \mathrm{~mm} / \mathrm{año}$ ), para desaguar en el río Paraná Medio a través del río Corrientes. 
La cuenca del Iberá se ha desarrollado sobre una base de arenas que corresponden al Plioceno Superior-Pleistoceno Inferior (Castellanos, 1965) en el margen occidental, mientras que en el margen oriental la superficie de la cubeta corresponde a una base de limo y arcilla en los primeros $50 \mathrm{~cm}$ (Iriondo, 1994). El fondo de la mayoría de los lagos del Iberá está cubierto por sedimentos arenosos, que serían relictos del modelado fluvial (Popolizio, 1981), como también ondulaciones hoy sumergidas bajo la vegetación, sin embargo, en la parte este de la laguna Iberá, los sedimentos son limo-arenosos, hasta limosos en algunos sectores.

Cuadrado y Neiff (1993) y posteriormente Fernandez-Pacella, Garralla, y Anzótegui (2011) efectuaron los primeros estudios polínicos en el Iberá; Fernandez-Pacella et al. sobre muestras de lagunas ubicadas en el noroeste del sistema, mientras que Cuadrado y Neiff sobre varias muestras de embalsados provenientes de la parte este del sistema, estos últimos sólo hallaron polen en los primeros metros del embalsado, y muy poco o ninguno en las muestras más profundas, también indicaron la ausencia de esporas fúngicas. Dada la amplia diversidad de las comunidades tropicales en la Provincia de Corrientes, es importante documentar, a través de floras polínicas, la morfología de las distintas especies que las conforman.

El género Senna forma parte de la subfamilia Caesalpinioideae, compuesta por 160 géneros y aproximadamente 1900 especies. En Argentina dicha subfamilia cuenta con 16 géneros y 83 especies, de los cuales se encuentran en el nordeste 11 géneros y 45 especies (Fernandez-Pacella, Anzótegui, \& Horn, 2010). La subfamilia está compuesta mayoritariamente por árboles y sub-arbustos y en menor medida por hierbas trepadoras. Se halla muy bien representada en regiones tropicales de América y África. Los géneros más numerosos son Senna (con 332 especies), Chamaecrista L. Moench (con 265) y Caesalpinia L. (con 100). Se encuentran en esta subfamilia, las casias, cina-cina, pata de vaca o pata de buey (Bauhinia L.), acacia negra (Gleditsia L.), guayacán (Caesalpinia gillesii (Wall. ex Hook.) Wall. ex D.Dietr.). Varias especies de cesalpinóideas son melíferas y otras se cultivan con fines decorativos, forestales, industriales o medicinales (Palacios-Chávez, Arreguín-Sánchez, de la Luz, \& Quiroz-García, 1996).

Una limitación importante para la correcta interpretación del impacto de las variaciones ambientales en la vegetación, ya sean climáticas y/o antropogénicas, es el conocimiento de la morfología polínica de las especies tropicales. Por lo tanto, se debe contar con una base de datos palinológico a nivel morfológico, que ayude a la interpretación y determinación de especies vegetales. Por lo anterior, el objetivo de este estudio fue describir la morfología polínica de las especies del género Senna Mill. de la zona Sureste del sistema Iberá, el género más representado de esta subfamilia en el Nordeste argentino.

\section{MATERIALES Y MÉTODOS}

Se elaboró un listado de especies pertenecientes a la selva del Nordeste argentino, más precisamente de la Provincia de Corrientes. Este listado se realizó mediante recolectas botánicas realizadas en los caminos y senderos de la zona núcleo (Sureste del Iberá), durante 5 a 8 días de cada mes, y abarcó el período entre 2009 y 2011. La recolecta botánica se realizó en bosques conservados y bosques que presentaron diferentes etapas de recuperación. Adicionalmente, se realizó una revisión de los ejemplares con base en literatura especializada (Abbiatti, 1946; Arbo \& Tressens, 1997, 2002; Carnevalli, 1994; Dimitri, 1974; Graham \& Barker, 1981; Fernandez-Pacella, et al., 2010, 2011) y bases de datos de herbario del Instituto de Botánica del Nordeste (IBONE). La recolecta y procesamiento de los ejemplares botánicos se realizó de acuerdo con la propuesta de Lot y Chiang (1986). Los nombres taxonómicos fueron corroborados en la base de datos del Missouri Botanical Graden W3TROPICOS (www.tropicos.org) consultado en el periodo 2009-2011).

En cuanto al estudio morfológico del polen se tomaron manualmente los botones florales, 
como mínimo tres de cada flor, obtenidos de ejemplares en la zona de estudio, para aquellas especies que no pudieron ser muestreadas en campo, se precisó de la toma de botones florales del herbario del IBONE. La preparación del material palinológico, se llevó a cabo por la técnica de acetólisis de Erdtman (1969) modificada. A fin de obtener resultados confiables, de cada muestra se contó como mínimo 300 granos de polen. La observación y microfotografias del material polínico se realizó con un fotomicroscopio Nikon Eclipse (E100) y Microscopio Electrónico de Barrido (MEB). Para realizar las descripciones de las diferentes especies se basó en la terminología de Erdtman (1969), complementándose con Kremp (1965), Punt, Hoen, Blackmore, Nilsson, y Le Thomas (2007), Roubik y Moreno (1991), considerando los siguientes caracteres morfológicos del polen: a) tipo, número y posición de las aberturas; b) descripción detallada de las aberturas, considerando la forma, estructura, tamaño y posición; c) exina: estructura y ornamentación señalando el tipo, la forma, la distribución de los elementos con el grosor de las capas (sexina; nexina); d) la asociación, simetría y polaridad (ASP); e) la forma del grano, indicando el contorno polar y ecuatorial; f) el tamaño en micras; g) índice del área polar o apocolpium (IAP); h) la forma de vida (FV) de la especie y finalmente información del ejemplar. En este trabajo se presentan 17 especies pertenecientes al género Senna, las cuales no se habían descrito anteriormente para el Iberá. Las preparaciones palinológicas fueron depositadas en la palinoteca del Centro de Ecología del Litoral (CECOAL) y en el herbario del Instituto de Botánica del Nordeste Argentino (IBONE).

\section{RESULTADOS}

El trabajo ha derivado en una lista de especies de plantas vasculares cercana a la realidad de la diversidad de especies que pudieran encontrarse en el Iberá y áreas aledañas. Las características topográficas y los accesos complicados restringieron la recolecta botánica en muchas zonas florísticamente importantes.
En este estudio se describen por primera vez para la zona, la morfología polínica de 17 especies, pertenecientes al género Senna, del sureste del sistema Iberá correspondientes a la familia Fabaceae, Subfamilia Caesalpinoideae. Desde el punto de vista palinológico el género está compuesto de granos 3-colporados pequeños a medianos, radiosimétricos, isopolares, oblatos-esferoidales a prolatos, ámbito subcircular, subtriangular a triangular; colpos largos y de ancho variable $5.8-15.8 \mu \mathrm{m}$, con costillas (en algunas especies); endoaperturas lalongadas de $6.3-16.4 \mu \mathrm{m}$ de ancho x 2.9-11.5 $\mu \mathrm{m}$ de largo o circulares, en algunas especies levemente sobreelevadas sobre la superficie del grano. Exina de 1.4-6.4 $\mu \mathrm{m}$ de espesor. El MEB revela que la sexina es tectada psilada, escabrada a rugulada; imperforada, punctada a perforada, en algunas especies se observa un pequeño anillo o fastigium externo en las endoaperturas y otras presentan nanoespínulas fuera del área polar.

A continuación se presentan las descripciones palinológicas ordenadas alfabéticamente por especies, de cada una de ellas se midieron los parámetros comúnmente utilizados en las descripciones palinológicas: diámetro polar, diámetro ecuatorial, espesor de la exina (sexina y nexina sólo en aquellas especies claramente distinguibles al MO), aperturas, elementos esculturales e índice del apocolpio (Cuadro 1).

\section{FABACEAE}

\section{Senna alata}

(L.) Roxb.

(Fig. 1A-C, F)

Abertura: Tricolporado. Colpos largos de $16.3-18 \mu \mathrm{m}, \mathrm{y} 13.4-15.8 \mu \mathrm{m}$ de ancho, endoaperturas lalongadas de 10-11.4 x 4.9-6.5 $\mu \mathrm{m}$ (ancho $\mathrm{x}$ largo), rodeada por un fastigium. Exina: tectada, rugulada, fosulada. Exina de $3.1-4 \mu \mathrm{m}$

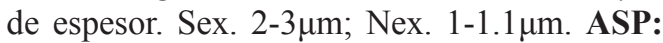
Mónada; isopolar; radiosimétrica. Forma: oblato; CP: subtriangular; CE: elíptico. Tamaño: $\mathrm{P}=18.9-20.3 \mu \mathrm{m} ; \mathrm{E}=30.8-32.2 \mu \mathrm{m}$. IAP: $6 \mu \mathrm{m}$ FV: arbusto o subarbusto. Ejemplar: Krapovikas y Cristobal 29073 (Ctes.). 
CUADRO 1

Lista de especies del género Senna con los valores de los principales caracteres analizados

TABLE 1

List of species of the genus Senna with the main characters found

\begin{tabular}{lccccccc}
\multicolumn{1}{c}{ Taxa } & $\mathrm{P}(\mu \mathrm{m})$ & $\mathrm{E}(\mu \mathrm{m})$ & $\mathrm{P} / \mathrm{E}$ & Ancho del colpo $(\mu \mathrm{m})$ & Endoapertura $(\mu \mathrm{m})$ & Exina $(\mu \mathrm{m})$ & $\mathrm{IAP}(\mu \mathrm{m})$ \\
Senna alata & $19(19) 20$ & $31(31) 32$ & 1.5 & $3-16$ & $14-16 \times 9-11$ & $3-4$ & 6 \\
S. chacoënsis & $19(20) 22$ & $22(23) 24$ & 1.0 & $6-9$ & $7-9$ & $1-3$ & 4 \\
S. chloroclada & $33(33) 34$ & $21(22) 24$ & 0.9 & $6-7$ & $6-8 \times 3-4$ & $2-3$ & 2.4 \\
S. corymbosa & $23(24) 26$ & $27(28) 29$ & 1.0 & $6-9$ & $7-9 \times 4-6$ & $3-4$ & 4.6 \\
S. hilariana & $27(28) 29$ & $23(23) 24$ & 1.0 & $6-8$ & $7-9 \times 4-5$ & $1-3$ & 6.7 \\
S. hirsuta & $31(32) 33$ & $23(24) 26$ & 1.3 & $7-8$ & $7-9$ & $2-3$ & 4.1 \\
S. morongii & $17(20) 23$ & $19(20) 21$ & 0.8 & $7-9$ & $7-9 \times 4-5$ & $1-3$ & 4.2 \\
S. nana & $31(32) 33$ & $29(30) 31$ & 1.0 & $7-8$ & $7-8$ & $2-4$ & 6.7 \\
S. oblongifolia & $19(20) 21$ & $23(24) 26$ & 1.3 & $7-8$ & $8-9 \times 4-4$ & $2-3$ & 4.2 \\
S. obtusifolia & $22(23) 24$ & $21(22) 23$ & 1.0 & $6-8$ & $7-9$ & $2-3$ & 4.8 \\
S. occidentalis & $34(35) 36$ & $31(31) 32$ & 1.0 & $6-8$ & $7-9$ & $3-5$ & 4.7 \\
S. pendula var. missionum & $22(23) 24$ & $24(24) 25$ & 1.0 & $6-8$ & $7-9$ & $3-5$ & 3.3 \\
S. pendula var. pendula & $26(27) 29$ & $20(21) 22$ & 0.9 & $6-8$ & $7-8 \times 3-4$ & $1-3$ & 4 \\
S. pilifera & $20(20) 21$ & $23(23) 24$ & 1.0 & $6-9$ & $7-9 \times 3-4$ & $2-3$ & 5 \\
S. scabriuscula & $33(34) 35$ & $31(31) 32$ & 1.0 & $7-8$ & $7-9$ & $3-6$ & 5.2 \\
S. spiniflora & $26(27) 28$ & $24(24) 25$ & 1.0 & $6-9$ & $7-9 \times 3-5$ & $2-4$ & 7.7 \\
S. spectabilis & $26(27) 29$ & $24(24) 25$ & 1.0 & $7-8$ & $7-9 \times 5-6$ & $2-3$ & 4.3 \\
\hline
\end{tabular}

\section{Senna chacoënsis}

(L. Bravo) H.S. Irwin \& Barneby

(Fig. 1D-E, I)

Abertura: Tricolporado. Colpos largos de $17.5-19.8 \mu \mathrm{m}$, у $6-9 \mu \mathrm{m}$ de ancho, endoaperturas circulares de 7-9.6 $\mu \mathrm{m}$ de diámetro. Exina: tectada, escabrada, imperforada. Exina de 1.4$2.8 \mu \mathrm{m}$ de espesor. Diferenciación entre sexina y nexina de difícil observación. ASP: Mónada; isopolar; radiosimétrica. Forma: oblato; CP: subtriangular; CE: elíptico. Tamaño: $\mathrm{P}=19.6-$ $21.7 \mu \mathrm{m} ; \mathrm{E}=22.4-23.8 \mu \mathrm{m}$. IAP: $4 \mu \mathrm{m} \quad \mathbf{F V}$ : arbusto o subarbusto. Ejemplar: Legname y Cuezzo 10452 (Ctes.).

\section{Senna chloroclada}

(Harms) H.S. Irwin \& Barneby.

(Fig. 1G-H, J)

Abertura: Tricolporado. Colpos largos de $25-29.6 \mu \mathrm{m}$, y $6.7-8 \mu \mathrm{m}$ de ancho, endoaperturas lalongadas de 6.3-7.7 x 3-4.5 $\mu \mathrm{m}$ (ancho x largo). Exina: tectada, rugulada, perforada. Exina de 2.1-3.5 $\mu \mathrm{m}$ de espesor. Sex. 1.3$2.5 \mu \mathrm{m}$; Nex. 0.8-1 $\mu \mathrm{m}$. ASP: Mónada; isopolar; radiosimétrica. Forma: prolato; CP: triangular;
CE: elíptico. Tamaño: $\mathrm{P}=32.9-34.3 \mu \mathrm{m} ; \mathrm{E}=21$ 24.5 $\mu \mathrm{m}$. IAP: $2.4 \mu \mathrm{m}$ FV: arbusto. Ejemplar: Krapovikas et al. 26714 (Ctes.).

\section{Senna corymbosa}

(Lam.) H.S. Irwin \& Barneby

(Fig. 2A-C)

Abertura: Tricolporado. Colpos largos de 17.1-19.4 $\mu \mathrm{m}$, y $6.5-8.7 \mu \mathrm{m}$ de ancho, endoapertura lalongadas de 7-9.1 x 4-6 $\mu \mathrm{m}$ (ancho $\mathrm{x}$ largo) rodeada por un anillo de sexina. Exina: tectada, rugulada, perforada. Exina de 2.8$3.5 \mu \mathrm{m}$ de espesor. Sex. 1.9-2.5 $\mu \mathrm{m}$; Nex. 0.9$1 \mu \mathrm{m}$. ASP: Mónada; isopolar; radiosimétrica. Forma: oblato; CP: subtriangular; CE: elíptico. Tamaño: $\mathrm{P}=23.1-25.9 \mu \mathrm{m} ; \mathrm{E}=27.3-29.4 \mu \mathrm{m}$. IAP: $4.6 \mu \mathrm{m}$ FV: arbusto o árbol. Ejemplar: Huidobro 3667 (Ctes.).

\section{Senna hilariana}

(Benth.) H.S. Irwin \& Barneby

(Fig. 2D-F)

Abertura: Tricolporado. Colpos largos de $21.8-23 \mu \mathrm{m}$, y $6.7-8 \mu \mathrm{m}$ de ancho, endoaperturas lalongadas de 7-8.9 x 3.9-4.6 $\mu \mathrm{m}$ (ancho $\mathrm{x}$ 

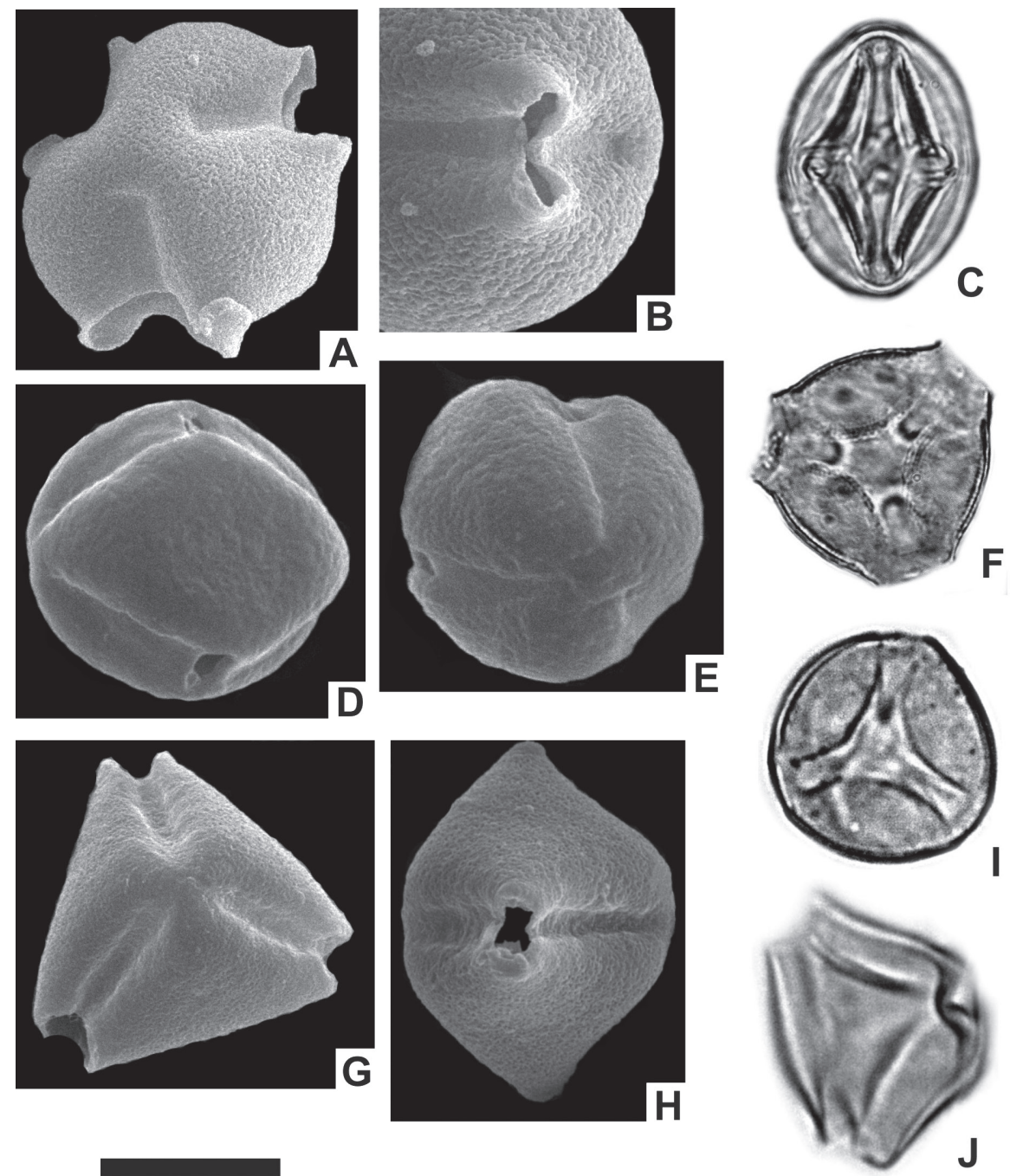

Fig. 1. Senna alata: A. vista polar (4 300x); B. detalle de la escultura fosulada-perforada y la endoapertura con anillo externo (10 000x); C, vista ecuatorial (100x); F. vista polar en foco superior (100x). Senna chacoënsis: D. vista ecuatorial (4 300x); E. vista polar (4 300x); I. vista polar, corte óptico (100x). Senna chlorochlada: G, vista polar (3 800x); H. vista ecuatorial (3 800x), en ambos se muestra la exina tectada, rugulada, perforada y endoaperturas sobreelevadas; J. vista polar (100x).

La escala equivale a $16.4 \mu \mathrm{m}$ en $\mathrm{A} ; 4 \mu \mathrm{m}$ en $\mathrm{B} ; 12.2 \mu \mathrm{m}$ en $\mathrm{C} ; 11 \mu \mathrm{m}$ en $\mathrm{D} ; 13.2 \mu \mathrm{m}$ en $\mathrm{E} ; 23.2 \mu \mathrm{m}$ en $\mathrm{F} ; 13 \mu \mathrm{m}$ en G; $21.2 \mu \mathrm{m}$ en $\mathrm{H} ; 17.2 \mu \mathrm{m}$ en I; $21 \mu \mathrm{m}$ en J.

Fig. 1. Senna alata: A. polar view (4 300x); B. detail of the fossulate-perforate sculpture and the aperture with external ring (10 000x); C, equatorial view (100x); F. polar view in top area (100x). Senna chacoënsis: D. equatorial view (4 300x); E. polar view (4 300x); I. polar view, optical cut (100x). Senna chlorochlada: G. polar view (3 800x); H. equatorial view (3 800x), in both it appears the tectate, rugulate, perforate exine and high aperture.

Scale bar represent $16.4 \mu \mathrm{m}$ in $\mathrm{A} ; 4 \mu \mathrm{m}$ in $\mathrm{B} ; 12.2 \mu \mathrm{m}$ in $\mathrm{C} ; 11 \mu \mathrm{m}$ in $\mathrm{D} ; 13.2 \mu \mathrm{m}$ in $\mathrm{E} ; 23.2 \mu \mathrm{m}$ in $\mathrm{F} ; 13 \mu \mathrm{m}$ in $\mathrm{G} ; 21.2 \mu \mathrm{m}$ in $\mathrm{H} ; 17.2 \mu \mathrm{m}$ in $\mathrm{I} ; 21 \mu \mathrm{m}$ in $\mathrm{J}$. 
largo), rodeada por un anillo de sexina. Exina: tectada, escabrada, punctada. Exina de 1.4$2.8 \mu \mathrm{m}$ de espesor. Sex. 0.8-2 $\mu \mathrm{m}$; Nex. 0.6$0.8 \mu \mathrm{m}$. ASP: Mónada; isopolar; radiosimétrica. Forma: prolato; CP: subtriangular; CE: elíptico. Tamaño: $\mathrm{P}=27.3-29.4 \mu \mathrm{m} ; \mathrm{E}=23.1-24.5 \mu \mathrm{m}$. IAP: $6.7 \mu \mathrm{m}$ FV: arbusto o subarbusto. Ejemplar: Schinini 8104 (Ctes.).

\section{Senna hirsuta}

(L.) H.S. Irwin \& Barneby

(Fig. 2G-I)

Abertura: Tricolporado. Colpos largos de $26.4-28.1 \mu \mathrm{m}$, у $6.9-8.4 \mu \mathrm{m}$ de ancho, endoaperturas circulares de 7.7-9.1 $\mu \mathrm{m}$ de diámetro. Exina: tectada, escabrada e imperforada. Exina de 2.1-3.5 $\mu \mathrm{m}$ de espesor. Sex. 1.1-2.5 $\mu \mathrm{m}$; Nex. 1-1 $\mu \mathrm{m}$. ASP: Mónada; isopolar; radiosimétrica. Forma: prolato; CP: subcircular; CE: elíptico. Tamaño: $\mathrm{P}=30.8-32.9 \mu \mathrm{m} ; \mathrm{E}=23.1-25.9 \mu \mathrm{m}$. IAP: $4.1 \mu \mathrm{m} \mathrm{FV:} \mathrm{hierba} \mathrm{o} \mathrm{subarbusto.} \mathrm{Ejem-}$ plar: Schinini 7996 (Ctes.).

\section{Senna morongii}

(Britton) H.S. Irwin \& Barneby

(Fig. 3A-C)

Abertura: Tricolporado. Colpos largos de $17-18.6 \mu \mathrm{m}$, y $6.7-8.8 \mu \mathrm{m}$ de ancho, endoaperturas lalongadas de 7-9.4 x 3.9-4.8 $\mu \mathrm{m}$ (ancho $\mathrm{x}$ largo). Exina: tectada, rugulada, punctada. Exina de 1.4-2.8 $\mu \mathrm{m}$ de espesor. Diferenciación entre sexina y nexina de difícil observación. ASP: Mónada; isopolar; radiosimétrica. Forma: prolato; CP: subtriangular; CE: elíptico. Tamaño: $\mathrm{P}=21.7-23.1 \mu \mathrm{m} ; \mathrm{E}=19.6-21 \mu \mathrm{m}$. IAP: $4.2 \mu \mathrm{m}$ FV: subarbusto. Ejemplar: Quarín y Tressens 1356 (Ctes.).

\section{Senna nana}

(Benth.) H.S. Irwin \& Barneby

(Fig. 3D-F)

Abertura: Tricolporado. Colpos largos de 27.3-29 $\mu \mathrm{m}$, y 7-7.6 $\mu \mathrm{m}$ de ancho, endoaperturas circulares de 7.4-8.3 $\mu \mathrm{m}$ de diámetro, rodeada por un anillo de sexina. Exina: tectada, rugulada, perforada a foveolada. Exina de 2.4-3.8 $\mu \mathrm{m}$ de espesor. Sex. 1.4-2.8 $\mu$ m; Nex. 1-1 $\mu$ m. ASP: Mónada; isopolar; radiosimétrica. Forma: subprolato-esferoidal; $\mathrm{CP}$ : subtriangular; $\mathrm{CE}$ : elíptico. Tamaño: $\mathrm{P}=31.5-32.9 \mu \mathrm{m} ; \mathrm{E}=29.4$ $31.5 \mu \mathrm{m}$. IAP: $6.7 \mu \mathrm{m}$ FV: hierba o subarbusto. Ejemplar: Krapovikas et al. 21196 (Ctes.).

\section{Senna oblongifolia}

(Vog.-Zuber) H.S. Irwin \& Barneby

(Fig. 3G-I)

Abertura: Tricolporado. Colpos largos de $15-17.2 \mu \mathrm{m}$, y $6.9-8.5 \mu \mathrm{m}$ de ancho, endoa-

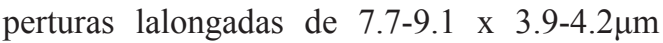
(ancho $\mathrm{x}$ largo), rodeada por un anillo de sexina. Exina: tectada, ligeramente rugulada, puctada a perforada. Exina de $2-3.4 \mu \mathrm{m}$ de espesor. Diferenciación entre sexina y nexina de difícil observación. ASP: Mónada; isopolar; radiosimétrica. Forma: oblato; $\mathrm{CP}$ : subtriangular a triangular; CE: elíptico. Tamaño: $\mathrm{P}=19.6-$ $21 \mu \mathrm{m} ; \mathrm{E}=23.1-25.9 \mu \mathrm{m}$. IAP: $4.2 \mu \mathrm{m} \quad \mathbf{F V}$ : arbusto. Ejemplar: Morrone et al.860 (Ctes.).

\section{Senna obtusifolia}

(L.) H.S. Irwin \& Barneby

(Fig. 4A-C)

Abertura: Tricolporado. Colpos largos de $14.1-16.1 \mu \mathrm{m}$, у $6.5-8.5 \mu \mathrm{m}$ de ancho, endoaperturas circulares de 7.1-9.1 $\mu \mathrm{m}$ de diámetro, rodeada por un anillo de sexina. Exina: tectada, rugulada, perforada. Exina de 2.1$3.5 \mu \mathrm{m}$ de espesor. Sex. 1.4-2.5 $\mu \mathrm{m}$; Nex. 0.7$1 \mu \mathrm{m}$. ASP: Mónada; isopolar; radiosimétrica. Forma: subprolato-esferoidal; CP: subtriangular-subcircular; CE: elíptico. Tamaño: $\mathrm{P}=22.4-$ $23.8 \mu \mathrm{m}$; $\mathrm{E}=21.7-23.1 \mu \mathrm{m}$. IAP: $4.8 \mu \mathrm{m}$ FV: hierba o subarbusto. Ejemplar: Fortunato 8063 (Ctes.).

\section{Senna occidentalis \\ (L.) Link \\ (Fig. 4D-F)}

Abertura: Tricolporado. Colpos largos de

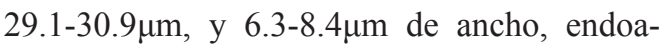
perturas circulares de $7-9 \mu \mathrm{m}$ de diámetro. Exina: tectada, rugulada, punctada. Exina de $3-5.4 \mu \mathrm{m}$ de espesor. Diferenciación entre sexina y nexina de difícil observación. ASP: Mónada; isopolar; radiosimétrica. Forma: prolato; CP: triangular; CE: elíptico. Tamaño: 

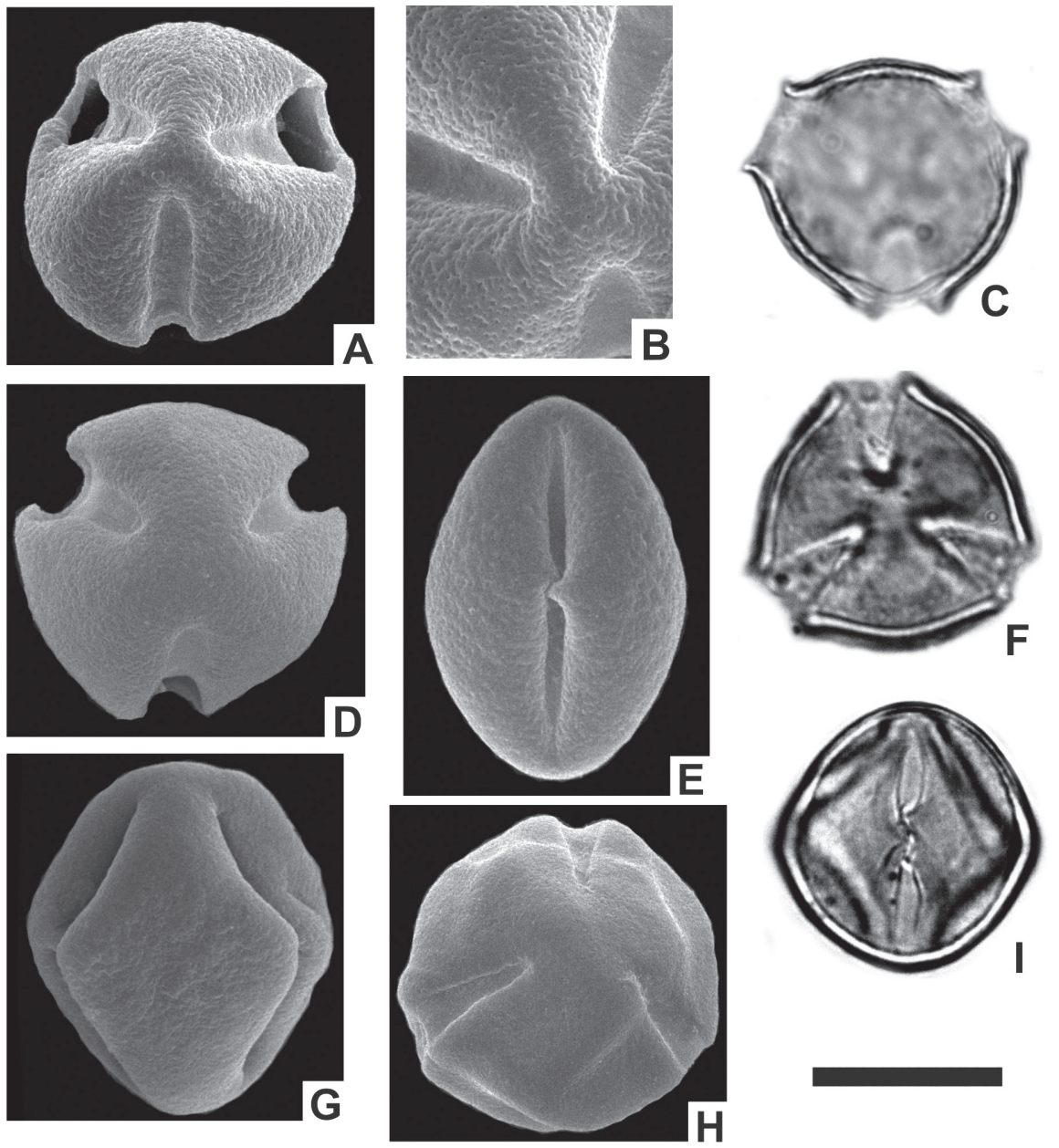

Fig. 2. Senna corymbosa: A. vista polar (4 300x); B. detalle de la escultura rugulada, perforada (10 000x); C. vista polar en corte óptico (100x). Senna hilariana: D. vista polar (4 000x); E. vista ecuatorial (4 000x), en ambas se observa la exina levemente escabrada, punctada; F. vista polar, corte óptico (100x). Senna hirsuta: G. vista ecuatorial (4 000x); H. vista polar (4 000x), en ambas se observa la exina levemente escabrada a psilada e imperforada; I. vista ecuatorial, corte óptico (100x).

La escala equivale a $15.7 \mu \mathrm{m}$ en $\mathrm{A} ; 4.5 \mu \mathrm{m}$ en B; $20 \mu \mathrm{m}$ en $\mathrm{C} ; 13 \mu \mathrm{m}$ en $\mathrm{D} ; 13.5 \mu \mathrm{m}$ en $\mathrm{E} ; 15.5 \mu \mathrm{m}$ en F; $16.6 \mu \mathrm{m}$ en G; $14 \mu \mathrm{m}$ en $\mathrm{H} ; 22 \mu \mathrm{m}$ en I.

Fig. 2. Senna corymbosa: A. polar view (4 300x); B. detail of the rugulate sculpture, perforate (10 000x); C. polar view in optical cut (100x). Senna hilariana: D. polar view (4000x); E, equatorial view (4000x), in both it appears the slightly scabrate, punctate exine; F. polar view, optical cut (100x). Senna hirsuta: G. equatorial view (4 000x); H. polar view (4 000x), in both it appears the slightly scabrate to psilate exine and imperforate; I. equatorial view, optical cut (100x). Scale bar represent $15.7 \mu \mathrm{m}$ in $\mathrm{A} ; 4.5 \mu \mathrm{m}$ in $\mathrm{B} ; 20 \mu \mathrm{m}$ in $\mathrm{C} ; 13 \mu \mathrm{m}$ in $\mathrm{D} ; 13.5 \mu \mathrm{m}$ in $\mathrm{E} ; 15.5 \mu \mathrm{m}$ in $\mathrm{F} ; 16.6 \mu \mathrm{m}$ in $\mathrm{G} ; 14 \mu \mathrm{m}$ in $\mathrm{H} ; 22 \mu \mathrm{m}$ in I. 

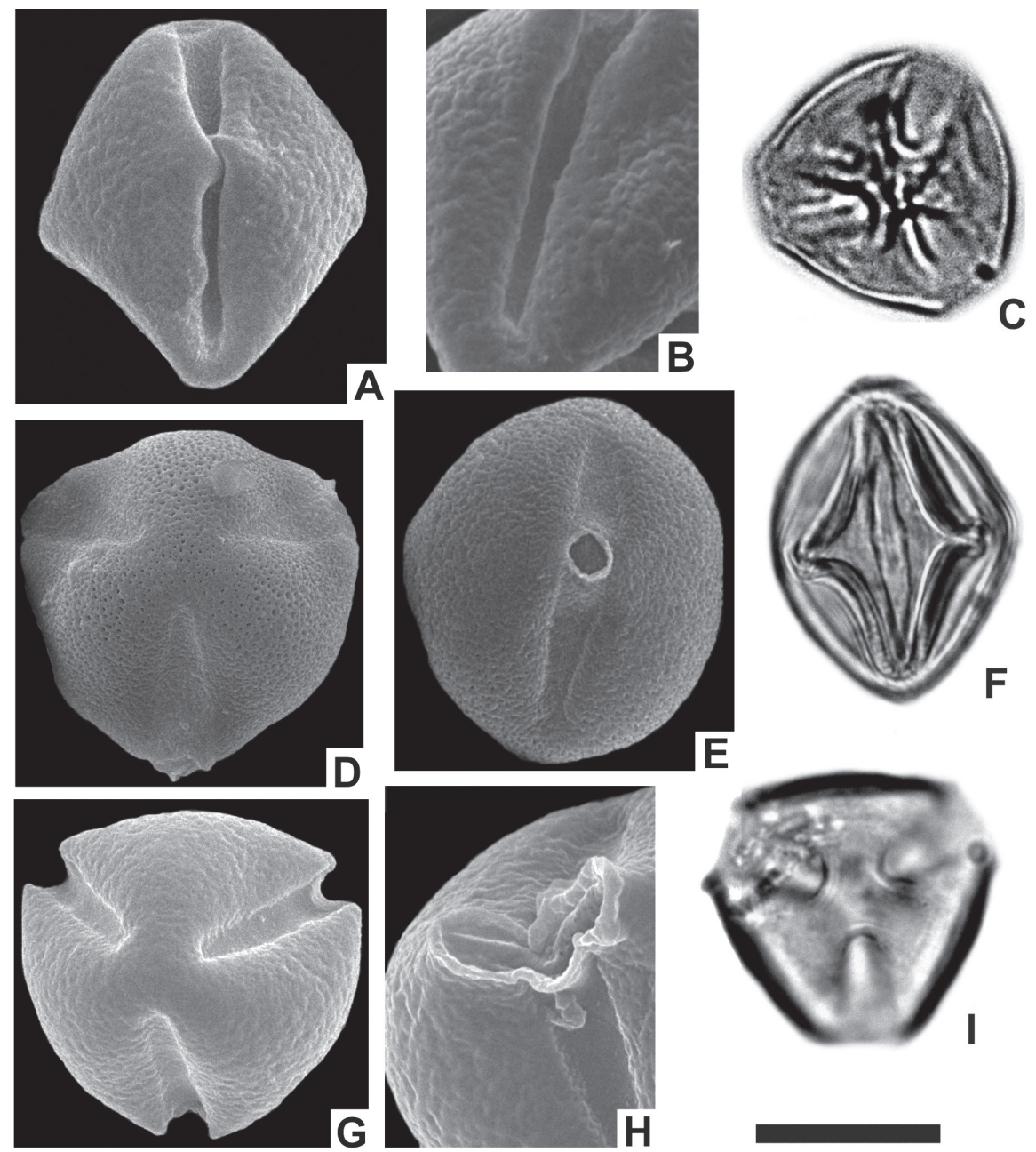

\section{$\mathrm{E}$}

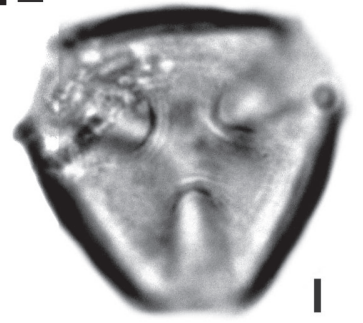

Fig. 3. Senna morongii: A. vista ecuatorial (4 300x); B. detalle de la escultura suavemente rugulada, punctada (10000x); C. vista polar, foco superior (100x). Senna nana: D. vista polar (3 800x); E. vista ecuatorial (3 800x); F. vista ecuatorial, foco medio superior (100x). Senna oblongifolia: G. vista polar (4 300x); H. detalle de la endoapertura anillada (10000x); I. vista polar, foco superior (100x).

La escala equivale a $10 \mu \mathrm{m}$ en $\mathrm{A} ; 2.7 \mu \mathrm{m}$ en $\mathrm{B} ; 14.2 \mu \mathrm{m}$ en $\mathrm{C} ; 16.6 \mu \mathrm{m}$ en $\mathrm{D} ; 16 \mu \mathrm{m}$ en $\mathrm{E} ; 18 \mu \mathrm{m}$ en $\mathrm{F} ; 13.2 \mu \mathrm{m}$ en $\mathrm{G} ; 3.4 \mu \mathrm{m}$ en $\mathrm{H} ; 16.2 \mu \mathrm{m}$ en $\mathrm{I}$.

Fig. 3. Senna morongii: A. equatorial view (4 300x); B. detail of the softly rugulate, punctate sculpture (10 000x); C. polar view, top area (100x). Senna nana: D. polar view (3 800x); E. equatorial view (3 800x); F. equatorial view, top way area (100x). Senna oblongifolia: G. polar view (4 300x); H. detail of the ringed aperture (10 000x); I. polar view, top area (100x). Scale bar represent $10 \mu \mathrm{m}$ in $\mathrm{A} ; 2.7 \mu \mathrm{m}$ in $\mathrm{B} ; 14.2 \mu \mathrm{m}$ in $\mathrm{C} ; 16.6 \mu \mathrm{m}$ in $\mathrm{D} ; 16 \mu \mathrm{m}$ in $\mathrm{E} ; 18 \mu \mathrm{m}$ in $\mathrm{F} ; 13.2 \mu \mathrm{m}$ in $\mathrm{G} ; 3.4 \mu \mathrm{m}$ in $\mathrm{H}$; $16.2 \mu \mathrm{m}$ in I. 
$\mathrm{P}=34.3-36.4 \mu \mathrm{m} ; \mathrm{E}=30.8-32.2 \mu \mathrm{m}$. IAP: $4.7 \mu \mathrm{m}$ FV: hierba. Ejemplar: Arbo 622 (Ctes.).

\section{Senna pendula}

(Willd.) H.S. Irwin \& Barneby var. missionum

(Fig. 4G-J)

Abertura: Tricolporado. Colpos largos de $17.1-19 \mu \mathrm{m}$, y $7-9 \mu \mathrm{m}$ de ancho, endoaperturas circulares de $7-9 \mu \mathrm{m}$ de diámetro, rodeada por un anillo de sexina. Exina: tectada, rugulada, perforada. Exina de 1.4-2.8 $\mu \mathrm{m}$ de espesor. Sex.

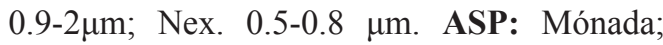
isopolar; radiosimétrica. Forma: suboblato; CP: subtriangular; CE: elíptico. Tamaño: $\mathrm{P}=22.4-23.8 \mu \mathrm{m} ; \mathrm{E}=23.8-25.2 \mu \mathrm{m}$. IAP: $3.3 \mu \mathrm{m}$ FV: subarbusto. Ejemplar: Tressens et al. 6637(Ctes.).

\section{Senna pendula}

(Willd.) H.S. Irwin \& Barneby var. pendula

(Fig. 5A-C)

Abertura: Tricolporado. Colpos largos de

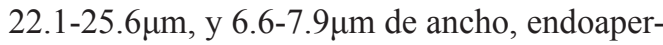
turas lalongadas de 7.1-8.6 x 3-4.5 $\mu \mathrm{m}$ (ancho $\mathrm{x}$ largo), rodeada por un anillo de sexina. Exina: tectada, rugulada, punctada. Exina de 1.4-2.8 $\mu \mathrm{m}$ de espesor. Sex. 1-1.8 $\mu \mathrm{m}$; Nex. 0.4$1 \mu \mathrm{m}$. ASP: Mónada; isopolar; radiosimétrica. Forma: prolato; CP: subtriangular; CE: elíptico. Tamaño: $\mathrm{P}=26.6-29.4 \mu \mathrm{m} ; \mathrm{E}=20.3-21.7 \mu \mathrm{m}$. IAP: $4 \mu \mathrm{m}$ FV: arbusto. Ejemplar: Dematteis et al. 930 (Ctes.).

\section{Senna pilifera}

(Vog.-Zuber) H.S. Irwin \& Barneby

(Fig. 5D-E)

Abertura: Tricolporado. Colpos largos de 25.1-29.3 $\mu \mathrm{m}$, y $6.4-8.7 \mu \mathrm{m}$ de ancho, endoaperturas lalongadas de 7.4-9.2 x 3.1-4.6 $\mu \mathrm{m}$ (ancho $\mathrm{x}$ largo), rodeada por un anillo de sexina. Exina: tectada, rugulada, perforada, presentando nanoespínulas fuera del área polar. Exina de 2.1-3.5 $\mu \mathrm{m}$ de espesor. Diferenciación entre sexina y nexina de difícil observación. ASP: Mónada; isopolar; radiosimétrica. Forma: prolato; CP: subtriangular; CE: elíptico. Tamaño: $\mathrm{P}=28.3-32.7 \mu \mathrm{m} ; \mathrm{E}=23.1-24.5 \mu \mathrm{m}$. IAP: $5 \mu \mathrm{m}$
FV: hierba o subarbusto. Ejemplar: Schinini et al. 18969 (Ctes.).

\section{Senna scabriuscula}

(Vog.-Zuber) H.S. Irwin \& Barneby

(Fig. 5F-H)

Abertura: Tricolporado. Colpos largos de $28.2-31 \mu \mathrm{m}$, y $6.8-8.4 \mu \mathrm{m}$ de ancho, endoaperturas circulares de $7-8.7 \mu \mathrm{m}$ de diámetro. Exina: tectada, rugulada, perforada. Exina de 3.5-6.1 $\mu \mathrm{m}$ de espesor. Diferenciación entre sexina y nexina de difícil observación. ASP: Mónada; isopolar; radiosimétrica. Forma: prolato; CP: subtriangular; CE: elíptico. Tamaño: $\mathrm{P}=33.6-35 \mu \mathrm{m} ; \quad \mathrm{E}=30.8-32.2 \mu \mathrm{m}$. IAP: $5.2 \mu \mathrm{m}$ FV: hierba o subarbusto. Ejemplar: Carnevalli 3260 (Ctes.).

\section{Senna spectabilis}

(D.C.) H.S. Irwin \& Barneby

(Fig. 6A-B)

Abertura: Tricolporado. Colpos largos de $22.4-24.7 \mu \mathrm{m}$, y $7-8.6 \mu \mathrm{m}$ de ancho, endoaperturas lalongadas de 7.4-8.9 x 4.9-6 $\mu \mathrm{m}$ (ancho $\mathrm{x}$ largo). Exina: tectada, levemente rugulada, perforada. Exina de 2.1-3.5 $\mu \mathrm{m}$ de espesor. Diferenciación entre sexina y nexina de difícil observación. ASP: Mónada; isopolar; radiosimétrica. Forma: prolato; $\mathrm{CP}$ : triangular; CE: elíptico. Tamaño: $\mathrm{P}=26.6-28.7 \mu \mathrm{m}$; $\mathrm{E}=23.8-25.2 \mu \mathrm{m}$. IAP: $7.7 \mu \mathrm{m}$ FV: arbusto o árbol. Ejemplar: Solomon 7350 (Ctes.).

\section{Senna spiniflora}

(Burkart) H.S. Irwin \& Barneby

(Fig. 6C-D)

Abertura: Tricolporado. Colpos largos de 23.9-25.6 $\mu \mathrm{m}$, y $6.5-9 \mu \mathrm{m}$ de ancho, endoaperturas lalongadas de 7-9.4 x 2.9-4,8 $\mu$ m (ancho x largo), rodeada por un anillo de sexina. Exina: tectada, rugulada, perforada. Exina de $2.1-4 \mu \mathrm{m}$ de espesor. Diferenciación entre sexina y nexina de difícil observación. ASP: Mónada; isopolar; radiosimétrica. Forma: prolato; CP: subtriangular; CE: elíptico. Tamaño: $\mathrm{P}=26.6-28 \mu \mathrm{m}$; $\mathrm{E}=23.8-25.2 \mu \mathrm{m}$. IAP: $4.3 \mu \mathrm{m}$ FV: arbusto. Ejemplar: Schinini y Pire 24928 (Ctes.). 

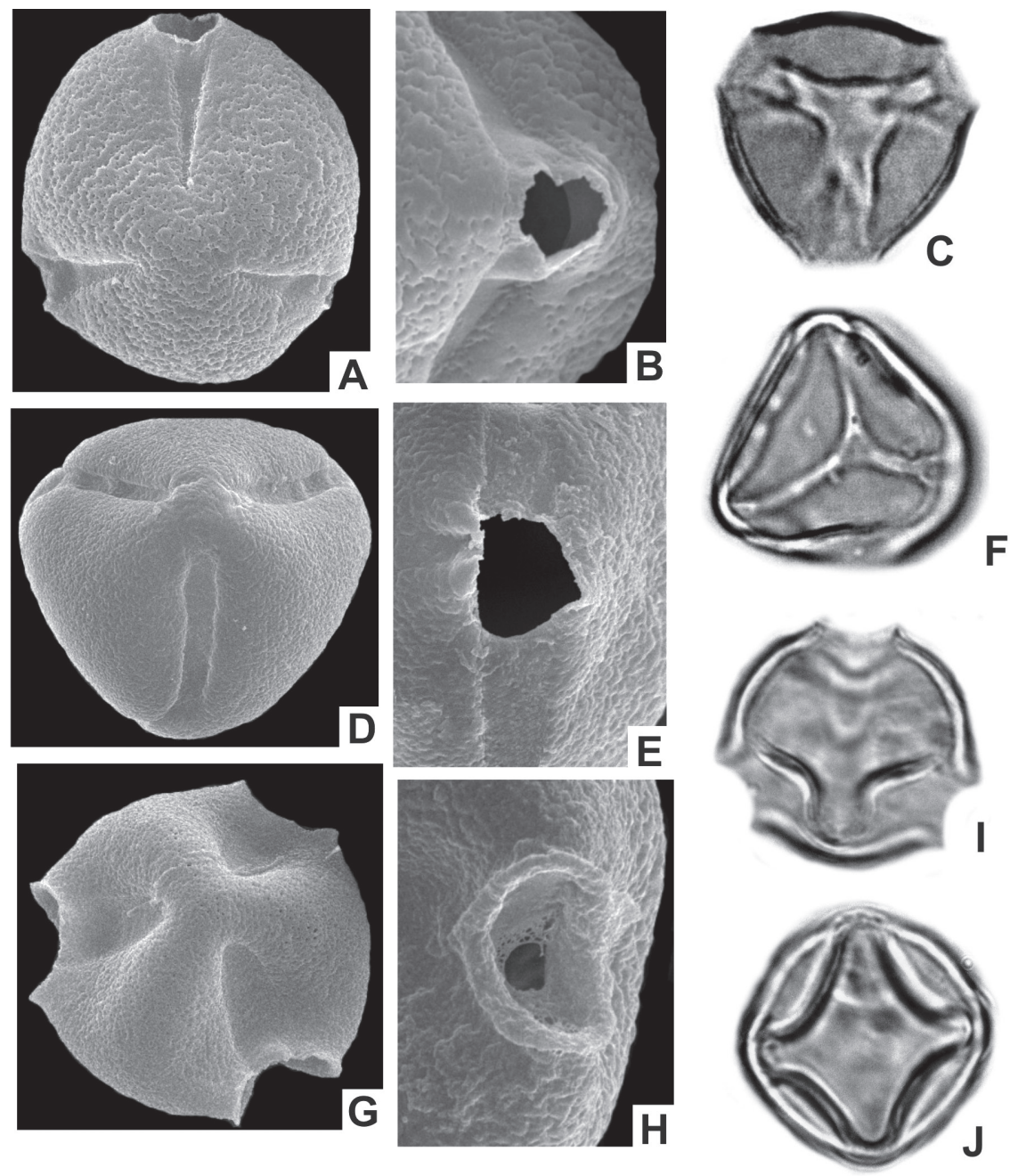

Fig. 4. Senna obtusifolia: A. vista polar (4 100x); B. detalle de la endoapertura circular anillada y de la exina tectada, rugulada, perforada (10 000x); C. vista polar (100x). Senna occidentalis: D. vista polar (3 700x); E. detalle de la exina tectada, rugulada, punctada y de la endoapertura circular $(10000 x)$; F. vista ecuatorial, foco superior (100x). Senna pendula var. missionum: G. vista polar (4 300x); H. detalle de la exina rugulada, perforada y de la endoapertura anillada y sobresaliente (10 000x); I. vista polar (100x); J. vista ecuatorial (100x), ambos en corte óptico.

La escala equivale a $11 \mu \mathrm{m}$ en A; $2.7 \mu \mathrm{m}$ en B y H; $18.5 \mu \mathrm{m}$ en C; $16 \mu \mathrm{m}$ en D; $4.5 \mu \mathrm{m}$ en E; $22 \mu \mathrm{m}$ en F; $13 \mu \mathrm{m}$ en G; $18 \mu \mathrm{m}$ en I; $15.5 \mu \mathrm{m}$ en J.

Fig. 4. Senna obtusifolia: A. polar view (4 100x); B. detail of the circular ringed aperture and the tectate, rugulate, perforate exine (10 000x); C. polar view (100x). Senna occidentalis: D. polar view (3 700x); E. detail of the tectate, rugulate, punctate exine and the circular aperture $(10000 x)$; F. equatorial view, top area $(100 x)$. Senna pendula var. missionum: G. polar view (4 300x); H. detail of the rugulate, perforate exine and the ringed and high aperture (10 000x); I. polar view (100x); J. equatorial view (100x), both in optical cut.

Scale bar represent $11 \mu \mathrm{m}$ in $\mathrm{A} ; 2.7 \mu \mathrm{m}$ in $\mathrm{B}$ y H; $18.5 \mu \mathrm{m}$ in $\mathrm{C} ; 16 \mu \mathrm{m}$ in $\mathrm{D} ; 4.5 \mu \mathrm{m}$ in $\mathrm{E} ; 22 \mu \mathrm{m}$ in $\mathrm{F} ; 13 \mu \mathrm{m}$ in $\mathrm{G} ; 18 \mu \mathrm{m}$ in I; $15.5 \mu \mathrm{m}$ in $\mathrm{J}$. 

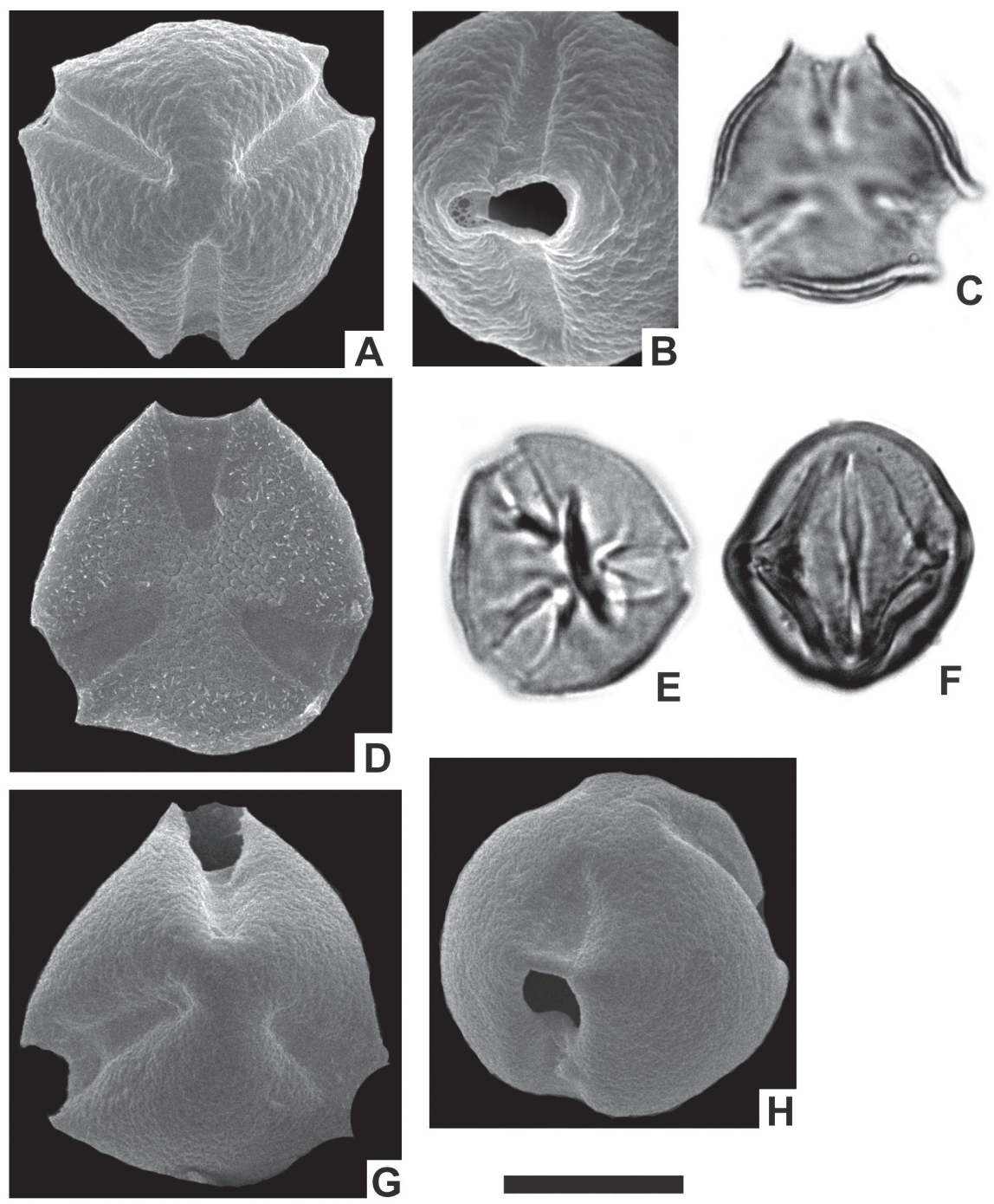

Fig. 5. Senna pendula var. pendula: A. vista polar (4 100x); B. detalle de la exina punctada y de la endoapertura anillada (10 000x); C. vista polar, corte óptico (100x). Senna pilifera: D. vista polar, mostrando la exina rugulada, perforada con nanoespínulas (3 800x); E. vista polar, foco superior (100x). Senna scabriuscula: F. vista ecuatorial, foco superior (100x); G. vista polar (3 800x); H. vista ecuatorial (3 800x); ambas muestran la exina tectada, rugulada, perforada; en H la endoapertura circular.

La escala equivale a $10.6 \mu \mathrm{m}$ en $\mathrm{A} ; 2.7 \mu \mathrm{m}$ en $\mathrm{B} ; 14 \mu \mathrm{m}$ en $\mathrm{C} ; 11.3 \mu \mathrm{m}$ en $\mathrm{D} ; 15.5 \mu \mathrm{m}$ en $\mathrm{E} ; 22 \mu \mathrm{m}$ en $\mathrm{F} ; 14.2 \mu \mathrm{m}$ en G; $17.3 \mu \mathrm{m}$ en $\mathrm{H}$.

Fig. 5. Senna pendula var. pendula: A. polar view (4 100x); B. detail of the punctate exine and the ringed aperture (10 000x); C. polar view, optical cut (100x). Senna pilifera: D. polar view, showing the rugulate, perforate exine with micro-thorns (3 800x); E. polar view, top area (100x). Senna scabriuscula: F. equatorial view, top area (100x); G. polar view (3 800x); H. equatorial view (3 800x); both appears the tectate, rugulate and perforate exine; in $\mathrm{H}$ the circular aperture.

Scale bar represent $10.6 \mu \mathrm{m}$ in $\mathrm{A} ; 2.7 \mu \mathrm{m}$ in $\mathrm{B} ; 14 \mu \mathrm{m}$ in $\mathrm{C} ; 11.3 \mu \mathrm{m}$ in $\mathrm{D} ; 15.5 \mu \mathrm{m}$ in $\mathrm{E} ; 22 \mu \mathrm{m}$ in $\mathrm{F} ; 14.2 \mu \mathrm{m}$ in $\mathrm{G} ; 17.3 \mu \mathrm{m}$ in $\mathrm{H}$. 


\section{DISCUSIÓN}

Como se observa en los resultados comentados anteriormente, las características morfológicas del género coinciden con lo expuesto por Buril, Alves, y Ribeiro dos Santos (2011), y Ventura y Huamán (2008) considerando al género Senna, como estenopolínico. La forma de las endoaperturas es el rasgo más importante que permite establecer dos tipos polínicos en los taxones estudiados.

- el Tipo I incluye las especies con endoaperturas lalongadas como Senna alata, $S$. chloroclada, S. corymbosa, S. hilariana,
S. morongii, S. oblongifolia, S. pendula var. pendula, S. pilifera, S. spiniflora y $S$. Spectabilis.

- el Tipo II incluye taxones con endoaperturas circulares como Senna chacoënsis, S. hirsuta, S. nana, S. obtusifolia, S. occidentalis, $S$. pendula var. missionum y $S$. scabriuscula.

En general, el tamaño de los granos de polen en Angiospermas parece ser un carácter con importante grado de variabilidad, dependiente de diversos factores del medio físico, como la nutrición mineral y/o la disponibilidad de agua (Muller, 1979; Ojeda \& Díez, 1992;
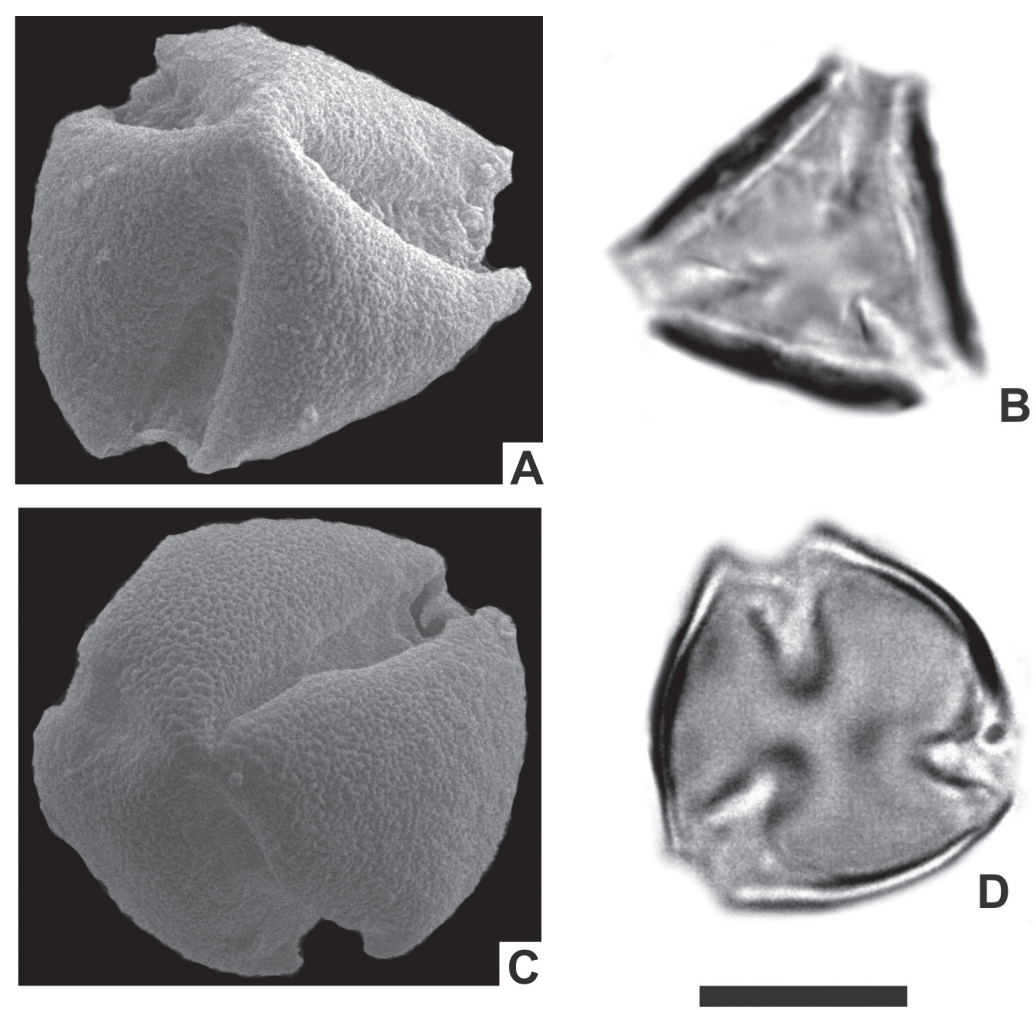

Fig. 6. Senna spectabilis: A. vista polar, se muestra la exina tectada rugulada, perforada (4 000x); B. vista polar en foco superior (100x). Senna spiniflora: C. vista polar mostrando la exina rugulada y perforada (4 000x); D. vista polar, corte óptico (100x).

La escala equivale a $10.6 \mu \mathrm{m}$ en $\mathrm{A} ; 15 \mu \mathrm{m}$ en $\mathrm{B} ; 10 \mu \mathrm{m}$ en $\mathrm{C} ; 13 \mu \mathrm{m}$ en D.

Fig. 6. Senna spectabilis: A. polar view, appears the tectate, rugulate and perforate exine (4 000x); B. polar view in top area (100x). Senna spiniflora: C. polar view, appears the rugulate and perforate exine (4 000x); D. polar view, optical cut (100x). Scale bar represent $10.6 \mu \mathrm{m}$ in $\mathrm{A} ; 15 \mu \mathrm{m}$ in $\mathrm{B} ; 10 \mu \mathrm{m}$ in $\mathrm{C} ; 13 \mu \mathrm{m}$ in D. 
Schoch-Bodmer, 1940; Walker \& Doyle, 1975; Walker, 1976) y las características climáticas (Lobreau-Callen, 1975). Las investigaciones paleoecológicas basadas en el análisis del contenido de polen en sedimentos de diferentes cuerpos de agua del Este del Iberá, han aportado datos sobre el impacto de los cambios climáticos y ambientales en la vegetación durante los últimos cientos de años (Fernandez-Pacella et al., 2011). El Iberá tiene áreas cuya vegetación original ha sido notablemente alterada y afectada, sin embargo, también tiene áreas como el Sureste y Suroeste, en varias etapas de sucesión (Fernandez-Pacella, 1013), por lo que se observa, desde el punto de vista fisonómico, el dominio de las especies herbáceas-arbustivas, mientras que las leñosas son muy escasas en cuanto a superficies parciales y totales. Por ello, el listado de especies pertenecientes al género Senna presentado en este trabajo, podría ser considerado en las actividades de restauración y recuperación de los diferentes tipos de vegetación.

Siendo el análisis de polen la herramienta para la reconstrucción de la historia de la vegetación, los conjuntos polínicos fósiles de zonas tropicales muestran que generalmente los granos de polen no son identificados a nivel específico, lo que lleva a la pérdida de información en las reconstrucciones paleo-ambientales. Adicionalmente, al ser una zona con una alta biodiversidad, se espera que este estudio sirva de base para futuras investigaciones sobre sistemática, historia de la vegetación y melisopalinología de esta región, ya que los sedimentos lacustres constituyen un medio ácido, pobre en oxígeno y favorable a la conservación de las esporas y de los granos de polen que se depositan en su superficie (Fernandez-Pacella et al., 2011).

\section{AGRADECIMIENTOS}

El autor desea expresar su agradecimiento al Instituto de Botánica del Nordeste (IBONE) por permitir observar la colección del herbario, al Prof. Oscar Canteros por realizar el procesamiento químico de las muestras y al Camping
Municipal de Colonia Carlos Pellegrini aprobando el acceso para recolectar material.

\section{RESUMEN}

El sistema de esteros y lagunas del Iberá, en la provincia de Corrientes abarcan aproximadamente $12000 \mathrm{~km}^{2}$ de superficie y es considerado uno de los humedales más importantes de Latinoamérica. Dada la gran diversidad de las comunidades tropicales en esta área, es importante generar nueva información sobre las diferentes especies de este ecosistema, a través de la morfología del polen, ya que los sedimentos lacustres constituyen un medio ácido, pobre en oxígeno y favorable a la conservación de granos de polen que se depositan en su superficie. Con el objetivo de promover futuras investigaciones sobre la sistemática, historia de la vegetación y melisopalinología de esta región, el estudio trató de describir la morfología polínica del género Senna, que se halla bien representado en la zona. Se elaboró un listado de especies pertenecientes a la Provincia de Corrientes. Este listado se realizó mediante recolectas botánicas realizadas en el Sureste del Iberá, durante 5 a 8 días de cada mes entre 2009 y 2011, adicionalmente, se consultó literatura especializada y bases de datos de herbario del Instituto de Botánica del Nordeste Argentino. En cuanto al estudio morfológico del polen, se tomaron manualmente los botones florales de ejemplares en la zona de estudio. Para aquellas especies que no pudieron ser muestreadas en campo, se precisó de la toma de botones florales del herbario del IBONE. La preparación del material palinológico se llevó a cabo por la técnica modificada de acetólisis de Erdtman. Las descripciones palinológicas consideran el diámetro polar y ecuatorial, grosor de la exina, aberturas y elementos esculturales. Los resultados muestran que el género Senna es euripolínico, compuesto de granos 3-colporados pequeños a medianos, radiosimétricos, isopolares, oblatos-esferoidales a prolatos, ámbito subcircular, subtriangular a triangular; colpos largos. Aquí, se describen por primera vez para la zona, la morfología polínica de 17 especies del género Senna, del sureste del sistema Iberá, reconociéndose dos grupos teniendo en cuenta endoaperturas: lalongadas y circulares. El listado de especies pertenecientes al género Senna presentado en este trabajo debe ser considerado en las actividades de restauración y recuperación de los diferentes tipos de vegetación. Una limitación importante para la correcta interpretación del impacto de las variaciones ambientales en la vegetación, ya sean climáticas y/o antropogénicas, es el conocimiento de la morfología polínica de las especies tropicales, por lo tanto, se debe contar con una base de datos palinológica a nivel morfológico que ayude a la interpretación y determinación de especies vegetales.

Palabras clave: morfología polínica de Senna, Sureste Iberá, diversidad, comunidades tropicales, paleoecológicas, Caesalpinioideae. 


\section{REFERENCIAS}

Abbiatti, D. (1946). Las Lornatáceas argentinas. Revista Museo La Plata, Sección Botánica, 7, 1-110.

Arbo, M. M. \& Tressens, S. G. (1997). Flora de la Reserva Natural Provincial del Iberá (Corrientes). Actas Ciencias Agrarias - Ciencias Biológicas, 3, 6-9.

Arbo, M. M. \& Tressens, S. G. (2002). Flora de Iberá. Corrientes, Argentina: EUDENE.

Buril, M. T.; Alves, M., \& Ribeiro dos Santos, Francisco de Assis (2011). Tipificação polínica em Leguminosae de uma área prioritária para conservação da Caatinga: Caesalpinioideae e Papilionoideae. Acta Botanica Brasilica, 25(3), 117-132.

Carnevalli, R. (1994). Fitogeografia de la Provincia de Corrientes. Asunción, Paraguay: LITOCOLOR.

Castellanos, A. (1965). Estudio fisiográfico de la provincia de Corrientes. Instituto de Fisiografía y Geología (Universidad Nacional del Litoral), 49, 100-222.

Cuadrado, G. A. \& Neiff, J. J. (1993). Palynology of Embalsados in Distrophic Lakes in Northeastern of Argentina. Revista Brasilera de Biología, 53, 443-451.

Dimitri, M. J. (1974). La Flora Arbórea del Parque Nacional Iguazú. Anales Parques Nacionales, 12, 27-180.

Erdtman, G. (1969). Handbook of Palynology: Morphology, taxonomy, ecology an introduction to the study of pollen grains and spores. New York: Hafner Publishing.

Fernandez-Pacella, L., Anzótegui, L. M., \& Horn, Y. M. (2010). Caesalpinoideae Fabaceae. In S. M. Pire, L. M. Anzótegui, \& G. A. Cuadrado (Eds.), Flora Polinica del Nordeste Argentino.(pp. 71-87). Corrientes, Argentina: EUDENE -UNNE.

Fernández-Pacella, L., Garralla, S., \& Anzótegui, L. M. (2011). Cambios en la vegetación durante el Holoceno en la región Norte del Iberá, Corrientes, Argentina. Revista de Biología Tropical, 59(1), 103-112.

Fernandez-Pacella, L. (2013). Palinología del Cuaternario en sedimentos lacustres del Noroeste del Iberá, Corrientes, Argentina. (Tesis Doctoral). Universidad Nacional del Nordeste, Corrientes. Argentina.

Graham, A. \& Barker, G. (1981). Palinology and Tribal Classification in the Caesalpinioidea. In R. M. Polhill, \& P. H Raven (Eds.), Advances in Legume Systematics (pp.802-833). Reino Unido: Royal Botanical Gardens Kew.

Iriondo, M. (1994). Los climas cuaternarios de la región Pampeana. Museo Provincial de Ciencias Naturales "Florentino Ameghino", 4, 481.
Kremp, G. O. W. (1965). Morphologic encyclopedia of palynology. Tucson: University of Arizona Press.

Lobreau-Callen, D. (1975) Les variations dimensionnelles du pollen du genre Ilex (Aquifoliaceae) et leurs rapports avec le climat. Bulletin de la Société Botanique de France, Collection Palynologie, 122, 179-199.

Muller, J. (1979) Form and function in Angiosperm pollen. Annals of the Missouri Botanical Garden, 66, 593-632.

Neiff, J. J. (1997). Ecología evolutiva del macrosistema Iberá (Corrientes, Argentina). (Tesis de Maestría). Universidad Nacional del Litoral, Santa Fe, Argentina.

Ojeda, F. \& Díez, M. J. (1992). Pollen dimorphism in three Teucrium species (Lamiaceae). Plant Systematics and Evolution, 183, 43-49.

Palacios-Chávez, R., Arreguín-Sánchez, M. de la Luz, \& Quiroz-García, D. L. (1996). Morfología de los granos de polen de la Subfamilia Caesalpinioideae (Leguminosae) del Valle de Mexico. Revista Polibotánica, 1, 16-21.

Popolizio, E. (1981). Diagnóstico del macrosistema del Iberá, Corrientes. Informe final del Convenio. Provincia de Corrientes (ICA)/INCYTH, 13 vol. Corrientes, Argentina.

Punt, W., Hoen, P. P., Blackmore, S., Nilsson, S., \& Le Thomas, A. (2007). Glossary of pollen and spore terminology. Review of Palaeobotany and Palynology, $143,1-81$.

Roubik, D. W. \& Moreno, J. E. (1991). Pollen and spores of Barro Colorado Island. Monographs in Systematic Botany, 36, 1-270.

Schoch-Bodmer, H. (1940). The influence of nutrition upon pollen-grain size in Lythrum salicaria. Journal of Genetics, 40, 393-402.

Ventura, K. \& Huamán, L. (2008). Morfología polínica de la familia Fabaceae de la parte baja de los Valles de Pativilca y Fortaleza (Lima-Perú). Biologist, 6(2), 112-134.

Walker, J. W. \& Doyle, J. A. (1975). The bases of Angiosperm phylogeny: Palynology. Annals of the Missouri Botanical Garden, 62, 664-723.

Walker, J. W. (1976). Evolutionary significance of the exine in the pollen of primitive Angiosperms. In I. K. Ferguson \& J. Moller (Eds.), The evolutionary significance of the exine. Linnean Society Symposium, 1, 251-308.

Tropicos.org. Missouri Botanical Garden. (2009-2011). Retrieved form http://www.tropicos.org 